\title{
How genome complexity can explain the difficulty of aligning reads to genomes
}

\author{
Vinhthuy Phan", Shanshan Gao, Quang Tran, Nam S Vo \\ From Fourth IEEE International Conference on Computational Advances in Bio and medical Sciences \\ (ICCABS 2014) \\ Miami Beach, FL, USA. 2-4 June 2014
}

\begin{abstract}
Background: Although it is frequently observed that aligning short reads to genomes becomes harder if they contain complex repeat patterns, there has not been much effort to quantify the relationship between complexity of genomes and difficulty of short-read alignment. Existing measures of sequence complexity seem unsuitable for the understanding and quantification of this relationship.

Results: We investigated several measures of complexity and found that length-sensitive measures of complexity had the highest correlation to accuracy of alignment. In particular, the rate of distinct substrings of length $k$, where $k$ is similar to the read length, correlated very highly to alignment performance in terms of precision and recall. We showed how to compute this measure efficiently in linear time, making it useful in practice to estimate quickly the difficulty of alignment for new genomes without having to align reads to them first. We showed how the lengthsensitive measures could provide additional information for choosing aligners that would align consistently accurately on new genomes.

Conclusions: We formally established a connection between genome complexity and the accuracy of short-read aligners. The relationship between genome complexity and alignment accuracy provides additional useful information for selecting suitable aligners for new genomes. Further, this work suggests that the complexity of genomes sometimes should be thought of in terms of specific computational problems, such as the alignment of short reads to genomes.
\end{abstract}

\section{Background}

Advances in next-generation sequencing technologies have driven the development of computational approaches to address the problem of aligning short reads to reference genomes [1-10]. Even so, the alignment problem remains challenging due to the presence of genomic repeats that are much longer than reads. Yu et al. [11] evaluated alignment performance of several aligners on repetitive regions selected from CpG islands and concluded that long repeats seriously degraded alignment performance.

Researchers generally believe that the difficulty of aligning short reads is very much related to the complexity of genomes; it is easier to misalign short reads when the genomes of interest have long and complicated repeat

Department of Computer Science, University of Memphis, Memphis, TN 38152, USA patterns. While there has been an interest in measuring complexity of strings, recent attention has been focused on complexity of DNA sequences [12-15]. Whiteford et al. [15] utilized k-mer frequencies as a way to visualize and understand the complexity of genomes. Kurtz et al. [14], similarly, annotated plant genomes with $k$-mer frequencies so that repeat structures and characteristics can be easily visualized. With the same approach to understanding genome complexity, Chor et al. [13] analyzed k-mer spectra of over 100 species and observed multimodal spectra for regions with specific CG content characteristics. Unfortunately, these measures cannot be easily quantified and immediately adopted to study how complexity affects the difficulty of short-read alignment.

In a recent study, Becher et al. [12] introduced a measure known as the $I$-complexity, which seems most promising as 
a tool to correlate sequence complexity to difficulty of short-read alignment. The authors showed several interesting properties of this measure, including its closeness to the Lempel-Ziv complexity and its efficient computation in linear time. The $I$-complexity can be easily adopted for our purpose and will be used among others to understand how genome complexity affects the difficulty of short-read alignment.

In this paper, we propose measures of complexity that are best suited for the analysis and understanding of the difficulty of short-read alignment and how such measures might be helpful in selecting appropriate aligners for new genomes. The inspiration for this work lies in the observation that complex repeat structures in DNA that affect the performance of computational tasks are length specific. For instance, in finding regulatory motifs in DNA sequences, repeated structures of interest are around 8-25 characters long. On the other hand, in aligning reads to genomes, such repeats probably have little effect on the performance of aligners. This means that measures such as the $I$-complexity that are general and not length-specific might not be best.

\section{Methods}

\section{I-complexity and $D$-complexity}

Becher et al. [12] introduced the I-complexity as a measure of complexity of strings. It is proportional to the number of distinct substrings of the input string. Specifically, the $I$-complexity of a DNA sequence $g$ is defined to be:

$$
I(g)=\sum_{i=1}^{|g|} \log _{4}(L C P[i]+2)-\log _{4}(L C P[i]+1)
$$

where $L C P[i]$ is the length of the longest common prefix of the suffixes of $g$ starting at positions $S[i]$ and at $S[i-1]$, and $S$ is the suffix array of $g$. The suffix array $S$ of $g$ stores implicitly lexicographically sorted suffixes of $g$; i.e. for $i<j, g_{S[i] \cdots|\ldots|}$ (the suffix starting at index $S[i]$ ) is lexicographically smaller than $g_{S[j] \cdots|g|}$ (the suffix starting at index $S[j])$.

The somewhat non-intuitive definition of the $I$-complexity has some advantages. The authors established upper and lower bounds for $I(g)$, and showed that it was close to the Lempel-Ziv complexity of $g$. Further, it can be computed in linear time because the suffix and $L C P$ arrays can be constructed in linear time [16,17].

Although the $I$-complexity will be used in our attempt to explore the relationship between complexity and difficulty of alignment, we introduce a similar measure, $D(g)$, counts directly the rate of distinct substrings:

$$
D(g)=\frac{2 \cdot|\{x: f(x)>0\}|}{|g| \cdot(|g|+1)}
$$

where $f(x)$ denotes the number of occurrences of $x$ in $g$. To be precise, $D(g)$ is equal to the total number of distinct substrings divided by the total number of substrings of $g$. $D(g)$ can be computed in linear time, due to the following lemma.

Lemma $1|\{x: f(x)>0\}|=\sum_{i=1}^{|g|} i-\sum_{i=1}^{|g|} L C P[i]$

Proof Suppose that a substring $s$ of $g$ occurs exactly $k$ times. Then, there will be a block of size $k$ in the suffix array that corresponds to $k$ suffixes that have $s$ as the common prefix. More specifically, assume that $s$ is the common prefix of the suffixes of $g$ starting at positions $S[i], S[i+1], \cdots, S[i+k-1]$. We will call the occurrence of $s$ at position $S[i]$ its representative occurrence, and its occurrences at $S[i+1], S[i+2], \cdots, S[i+k-1]$ its repeat occurrences.

Each repeat occurrence of $s$ is a prefix of the longest common prefix of the suffixes starting at $S[i+1], S[i+2], \cdots, S[i+k-1]$. This means, each repeat occurrence of $s$ is accounted for uniquely by the values of $L C P[i+1], L C P[i+2], \cdots, L C P[i+k-1]$.

If we focus on a position, for example $i+1$, we can see that the longest common prefix between $S[i+1]$ and $S[i]$ (let's call it $p_{1 \ldots j}$ ) accounts uniquely for $j$ repeat occurrences, namely $p_{1}, p_{1 \cdots 2}, \cdots, p_{1 \cdots(j-1)}$. One of these is $s$; the rest are repeat occurrences of other substrings. Thus, each repeat occurrence is accounted for uniquely in some entry of $L C P$ and each entry of $L C P$ accounts uniquely for some repeat occurrences. That implies that $\sum_{i=1}^{|g|} L C P[i]$ accounts for the total repeat occurrences of all substrings of $g$.

Further, $\sum_{i=1}^{|g|} i$ is the total number of substrings of $g$, since there are exactly $i$ substrings starting at position $i$. Thus, if we remove all repeat occurrences from the total number of substrings, we will get precisely the total number of representative occurrences. This means $|\{x: f(x)>0\}|=\sum_{i=1}^{|g|}-\sum_{i=1}^{|g|} L C P[i]$.

\section{Length-sensitive measures of complexity}

In addition to the $I$ and $D$, we introduce two notions of length-sensitive measures of genome complexity. The motivation is that, depending on which computational tasks that are affected by the complexity of genomes, only a narrow range of repeat lengths play an important role; for instance, one would expect repeats that affect the finding of regulatory motifs to be much shorter than those that affect the alignment reads to genomes. Given a number $k$, we define $D_{k}$ and $R_{k}$ as follows:

$$
\begin{aligned}
& D_{k}(g)=\frac{|\{x: f(x)>0 .|x|=k\}|}{|g|-k+1} \\
& R_{k}(g)=\frac{\sum_{f(x)>1,|x|=k} f(x)}{|g|-k+1}
\end{aligned}
$$


where $f(x)$ is the number of occurrences of $x$ in $g$. $D_{k}$ and $R_{k}$ measure the rates of distinct substrings and repeats, respectively, of length $k . R_{k}$ and $D_{k}$ are not exact "opposites" because $R_{k}$ does not account for nonrepeats, whereas $D_{k}$ does. $R_{k}$ is related to the function $C$ $(k, r)$ proposed by Whiteford et al. [15]. $C(k, r)$ is the count of $k$-mers repeating exactly $r$ times. Therefore, $R_{k}=\sum_{r>1} r \cdot C(k, r) . D_{k}$ and $R_{k}$ can be computed in linear time and space using suffix and LCP arrays.

Lemma $2|\{x: f(x)>0,|x|=k\}|=\mid\{j: s[j] \leq|g|-k+1$ and $L C P[j]<k\} \mid$

Proof A $k$-substring of $g$ must start at an index between 1 and $|g|-k+1$. Further, if $L C P[j]<k$, the $k$-prefix of the suffix starting at $S[j]$ is different from the $k$-prefix of the suffix starting at $S[j-1]$. Thus, each $j$ for which $S$ $[j] \leq|g|-k+1$ and $L C P[j]<k$ represents exactly one distinct $k$-substring.

On the other hand, if $S[j]>|g|-k+1$ or $L C P[j] \geq k$, then the $k$-substring starting at $S[j]$ does not exist or is not distinct. Since LCP runs through all positions of $g$, all distinct $k$-substrings are uniquely accounted for.

Lemma $3 \sum_{f(x)>1,|x|=k} f(x)=\sum_{|i, j| \in I_{k}}(j-i+2)$, where $I_{k}$ is the set of intervals $[i, j]$ 's, where $i \leq j$, such that

$$
\begin{aligned}
& 1 L C P[u] \geq k \text { for } i \leq u \leq j \\
& 2 L C P[i-1]<k \text { unless } i=1 \\
& 3 L C P[j+1]<k \text { unless } j=|g|
\end{aligned}
$$

Proof A $k$-repeat is a substring $x$ of length $k$, with $f(x)>1$. Since the suffix array $S$ is sorted lexicographically, $S$ forms consecutive runs of $k$-repeats, which are $k$-prefixes of the suffixes stored implicitly by $S$. More specifically, each interval $[i, j] \in I_{k}$ corresponds to all occurrences of exactly one $k$-repeat. The number of occurrences for each $k$-repeat is exactly $j-i+2$.

$I_{k}$ can be computed in linear time by scanning the $L C P$ array once. Note that the index of LCP runs from 1 to $|g|$, and $L C P[1]=0$.

\section{Relating genome complexity to difficulty of aligning short reads to genomes}

$I, D, D_{k}$, and $R_{k}$ provide quantitative measures of complexity for each genome. Intuitively, the more distinct substrings a reference genome has (i.e. high values of $I, D$, and $D_{k}$ ), the easier to align reads to the reference genome. Conversely, the more long repeats the genome has, the more difficult to align reads to it correctly (since the probability of mismatching of a read with a wrong substring is higher.)

We measure the performance of an alignment algorithm using precision and recall, where precision is defined as the fraction of aligned reads that are correct (i.e. number of correctly aligned reads divided by the total number of aligned reads); and recall is defined as the fraction of reads that are correctly aligned (i.e. number of correctly aligned reads divided by the total number of reads). These definitions were also used by Liu et al. [8].

To correlate complexity values to difficulty of alignment, for each measure of complexity, we computed the linear correlation between the complexity values of sequences in a diverse data set including 100 genomic sequences, and the actual performance for each of 10 popular aligners. A good measure of complexity will correlate highly with alignment performance.

\section{Results}

\section{Aligners and genomic data}

We selected from NCBI and EMBL-EBI databases a total of 100 genomic sequences from bacteria, plants, and eukaryotes (including human chromosomes) with diverse complexity. Detail information of these sequences is described in Tables 1, 2, and 3. " $\mathrm{N}$ " bases were removed from these genomic sequences because they were not real contents and constituted false long repeats that inappropriately affected the true complexity of the genomes.

We selected 10 popular short-read aligners that employ different algorithmic techniques and indexing structures: SHRiMP2 [1], mrFAST [2], SeqAlto [3], GASSST [4], Bowtie2 [5], BWA-SW [6], SOAP2 [7], CUSHAW2 [8], Masai [9], and Smalt [10].

We used default parameters to run these programs because these aligners appeared to perform well and consistent over the 100 genomes at such settings.

It is not possible to compute the number of correctly aligned reads for real reads because positions of real reads in reference genomes are not known. Consequently, precision and recall cannot be computed using real reads. For this reason, we simulated reads for each genome, $2 x$ coverage of reads at lengths 50,75 , and 100 using the wgsim program [18]. Reads were generated with default parameters; sequencing error rates equal to $0.5 \%, 1 \%, 2 \%$, and mutation rates between $0.1 \%$ and $1 \%$, of which $15 \%$ are indels. These parameters should be sufficiently realistic for the current sequencing technologies and a large range of organisms.

\section{Overview performance of aligners}

Figure 1 compares the running times of the aligners as a function of genome size (with $2 x$ coverage). To take advantage of multiple CPU cores, one could manually partition reads into separate sets and run multiple instances across the number of cores. But since some of the aligners were not designed for multiple cores, it made more sense to compare them in single-threaded mode. We found that SHRiMP2 was roughly a magnitude slower than the fastest aligners for larger genomes. Therefore, it was therefore excluded from the figure. 
Table 1. Information on the selected 100 genomic sequences [Part 1]

\begin{tabular}{|c|c|c|c|c|}
\hline ID & Genome size & Description & Lineage & Source \\
\hline AE017198 & 1992676 & Lactobacillus johnsonii NCC 533, & Bacteria, Firmicutes & $\mathrm{EB} \mid$ \\
\hline AJ270060 & 14497843 & Arabidopsis thaliana DNA chr. 4, long arm & Eukaryota, Viridiplantae & $\mathrm{EB} \mid$ \\
\hline AM055943 & 2013089 & Toxoplasma gondii RH, genomic DNA chr. Ib & Eukaryota, Alveolata & $\mathrm{EB} \mid$ \\
\hline AM263198 & 2814130 & Listeria welshimeri serovar 6b str. SLCC5334 & Bacteria, Firmicutes & $\mathrm{EB} \mid$ \\
\hline AM269894 & 1347714 & Eimeria tenella chr. 1, ordered contigs & Eukaryota, Alveolata & $\mathrm{EB} \mid$ \\
\hline BA000004 & 4202352 & Bacillus halodurans C-125 DNA, & Bacteria, Firmicutes & $\mathrm{EBI}$ \\
\hline BN001302 & 4011161 & TPA: Aspergillus nidulans FGSC A4 chr. II & Eukaryota, Fungi & $\mathrm{EB} \mid$ \\
\hline BX284601 & 15072434 & Caenorhabditis elegans Bristol N2 genomic chr., I & Eukaryota, Metazoa & $\mathrm{EBI}$ \\
\hline CAID01000012 & 521582 & Ostreococcus tauri WGS project CAID00000000 data, contig chr. 12 & Eukaryota, Viridiplantae & $\mathrm{EB} \mid$ \\
\hline CM000001 & 122678785 & Canis lupus familiaris chr. 1 & Eukaryota, Metazoa & $\mathrm{EB} \mid$ \\
\hline CM000038 & 23914537 & Canis lupus familiaris chr. 38 & Eukaryota, Metazoa & $\mathrm{EB} \mid$ \\
\hline CM000043 & 1786351 & Cryptococcus neoformans var. neoformans B-3501A chr. 4 & Eukaryota, Fungi & $\mathrm{EB} \mid$ \\
\hline CM000071 & 19787792 & Drosophila pseudoobscura pseudoobscura strain MV2-25 chr. 3 & Eukaryota, Metazoa & $\mathrm{EB} \mid$ \\
\hline CM000091 & 57791882 & Rattus norvegicus strain BN/SsNHsdMCW chr. 20 & Eukaryota, Metazoa & $\mathrm{EB} \mid$ \\
\hline CM000110 & 11219875 & Gallus gallus chr. 18 & Eukaryota, Metazoa & $\mathrm{EBI}$ \\
\hline CM000134 & 21712932 & Oryza sativa (indica cultivar-group) chr. 9 & Eukaryota, Viridiplantae & $\mathrm{EBI}$ \\
\hline CM000152 & 6357299 & Dictyostelium discoideum AX4 chr. 3 & Eukaryota, Amoebozoa & $\mathrm{EBI}$ \\
\hline CM000157 & 22324452 & Drosophila yakuba strain Tai18E2 chr. $2 \mathrm{~L}$ & Eukaryota, Metazoa & $\mathrm{EBI}$ \\
\hline CM000158 & 21139217 & Drosophila yakuba strain Tai18E2 chr. 2R & Eukaryota, Metazoa & $\mathrm{EBI}$ \\
\hline CM000169 & 4918979 & Aspergillus fumigatus Af293 chr. 1 & Eukaryota, Fungi & $\mathrm{EB} \mid$ \\
\hline CM000177 & 161428367 & Bos taurus chr. 1 & Eukaryota, Metazoa & $\mathrm{EB} \mid$ \\
\hline CM000201 & 44081797 & Bos taurus chr. 25 & Eukaryota, Metazoa & $\mathrm{EB} \mid$ \\
\hline CM000208 & 4054025 & Trypanosoma brucei brucei strain 927/4 GUTat10.1 chr. 10 & Eukaryota, Euglenozoa & $\mathrm{EB} \mid$ \\
\hline CM000209 & 199526509 & Mus musculus chr. 1 & Eukaryota, Metazoa & $\mathrm{EB} \mid$ \\
\hline CM000302 & 78773432 & Macaca mulatta chr. 16 & Eukaryota, Metazoa & $\mathrm{EB} \mid$ \\
\hline CM000377 & 185838109 & Equus caballus chr. 1 & Eukaryota, Metazoa & $\mathrm{EB} \mid$ \\
\hline CM000452 & 2067354 & Plasmodium vivax chr. 11 & Eukaryota, Alveolata & $\mathrm{EBI}$ \\
\hline CM000515 & 118548696 & Taeniopygia guttata chr. 1 & Eukaryota, Metazoa & $\mathrm{EBI}$ \\
\hline CM000530 & 16962381 & Taeniopygia guttata chr. 13 & Eukaryota, Metazoa & $\mathrm{EB} \mid$ \\
\hline CM000572 & 46535552 & Pongo abelii chr. 22 & Eukaryota, Metazoa & $\mathrm{EB} \mid$ \\
\hline CM000575 & 8914601 & Fusarium graminearum $\mathrm{PH}-1$ chr. 2 & Eukaryota, Fungi & $\mathrm{EB} \mid$ \\
\hline CM000580 & 4643527 & Gibberella moniliformis 7600 chr. 3 & Eukaryota, Fungi & $\mathrm{EB} \mid$ \\
\hline CM000592 & 5212762 & $\begin{array}{l}\text { Fusarium oxysporum f. sp. Lycopersici } \\
4287 \mathrm{chr} .4\end{array}$ & Eukaryota, Fungi & $\mathrm{EB} \mid$ \\
\hline
\end{tabular}

Table 2. Information on the selected 100 genomic sequences [Part 2]

\begin{tabular}{|c|c|c|c|c|}
\hline ID & Genome size & Description & Lineage & Source \\
\hline CM000612 & 1002813 & $\begin{array}{l}\text { Phaeodactylum tricornutum CCAP } \\
1055 / 1 \text { chr. } 9\end{array}$ & Eukaryota, Stramenopiles & EBI \\
\hline CM000638 & 3042585 & $\begin{array}{l}\text { Thalassiosira pseudonana CCMP1335 } \\
\text { chr. } 1\end{array}$ & Eukaryota, Stramenopiles & $\mathrm{EB} \mid$ \\
\hline CM000692 & 1385275 & $\begin{array}{l}\text { Saccharomyces kluyveri NRRL Y-12651 } \\
\text { chr. F }\end{array}$ & Eukaryota, Fungi & EBI \\
\hline CM000767 & 55460251 & Sorghum bicolor chr. 8 & Eukaryota, Viridiplantae & $\mathrm{EB} \mid$ \\
\hline CM000769 & 60981646 & Sorghum bicolor chr. 10 & Eukaryota, Viridiplantae & $\mathrm{EB} \mid$ \\
\hline CM000777 & 301354135 & Zea mays chr. 1. & Eukaryota, Viridiplantae & $\mathrm{EB} \mid$ \\
\hline CM000799 & 47997241 & Oryctolagus cuniculus chr. 10 & Eukaryota, Metazoa & $\mathrm{EB} \mid$ \\
\hline CM000829 & 61220071 & Sus scrofa chr. 18. & Eukaryota, Metazoa & $\mathrm{EB} \mid$ \\
\hline CM000831 & 1255352 & $\begin{array}{l}\text { Drosophila virilis strain 15010-1051.88 } \\
\text { chr. } 6 .\end{array}$ & Eukaryota, Metazoa & $\mathrm{EB} \mid$ \\
\hline CM000850 & 41906774 & Glycine max chr. 17 & Eukaryota, Viridiplantae & EBI \\
\hline
\end{tabular}


Table 2. Information on the selected 100 genomic sequences [Part 2] (Continued)

\begin{tabular}{|c|c|c|c|c|}
\hline CM000875 & 44557958 & Callithrix jacchus chr. 20 & Eukaryota, Metazoa & $\mathrm{EBI}$ \\
\hline CM000906 & 55886266 & Ovis aries chr. 22 & Eukaryota, Metazoa & EBI \\
\hline CM000907 & 66770968 & Ovis aries chr. 23 & Eukaryota, Metazoa & EBI \\
\hline CM000917 & 27037145 & Nasonia vitripennis chr. 3 & Eukaryota, Metazoa & $\mathrm{EBI}$ \\
\hline CM001221 & 42630297 & Medicago truncatula chr. 5. & Eukaryota, Viridiplantae & EBI \\
\hline CM001222 & 23282162 & Medicago truncatula chr. 6. & Eukaryota, Viridiplantae & EBI \\
\hline CM001276 & 232296185 & Macaca fascicularis chr. 1 & Eukaryota, Metazoa & EBI \\
\hline CM001294 & 65364038 & Macaca fascicularis chr. 19 & Eukaryota, Metazoa & EBI \\
\hline СР000048 & 922307 & Borrelia hermsii DAH, & Bacteria, Spirochaetes & EBI \\
\hline CP000496 & 2740984 & Scheffersomyces stipitis CBS 6054 chr. 2, complete sequence. & Eukaryota, Fungi & $\mathrm{EBI}$ \\
\hline CP000828 & 6503724 & Acaryochloris marina MBIC11017, & Bacteria, Cyanobacteria & $\mathrm{EBI}$ \\
\hline CP001037 & 8234322 & Nostoc punctiforme PCC 73102, & Bacteria, Cyanobacteria & $\mathrm{EBI}$ \\
\hline CP001141 & 945026 & $\begin{array}{l}\text { Phaeodactylum tricornutum CCAP } \\
1055 / 1 \text { chr. } 11 \text {, complete sequence. }\end{array}$ & Eukaryota, Stramenopiles & $\mathrm{EBI}$ \\
\hline CP001681 & 5167383 & Pedobacter heparinus DSM 2366, & Bacteria, Bacteroidetes & $\mathrm{EBI}$ \\
\hline CP001699 & 9127347 & Chitinophaga pinensis DSM 2588, & Bacteria, Bacteroidetes & $\mathrm{EBI}$ \\
\hline CP001982 & 5097447 & Bacillus megaterium DSM319, & Bacteria, Firmicutes & EBI \\
\hline CP002287 & 7013095 & Achromobacter xylosoxidans A8, & Bacteria, Proteobacteria & $\mathrm{EBI}$ \\
\hline СР002987 & 4044777 & Acetobacterium woodii DSM 1030, & Bacteria, Firmicutes & $\mathrm{EBI}$ \\
\hline СР003170 & 9239851 & Actinoplanes sp. SE50/110, & Bacteria, Actinobacteria & $\mathrm{EBI}$ \\
\hline CP003348 & 4321753 & Desulfitobacterium dehalogenans ATCC 51507, & Bacteria, Firmicutes & EBI \\
\hline CP003872 & 5196935 & Acidovorax sp. KKS102, & Bacteria, Proteobacteria & EBI \\
\hline CR380954 & 1050361 & Candida glabrata strain CBS138 chr. H complete sequence. & Eukaryota, Fungi & $\mathrm{EBI}$ \\
\hline CU234118 & 7456587 & Bradyrhizobium sp. ORS278,complete sequence. & Bacteria, Proteobacteria & $\mathrm{EBI}$ \\
\hline CU329672 & 2452883 & Schizosaccharomyces pombe chr. III, complete sequence & Eukaryota, Fungi & EBI \\
\hline
\end{tabular}

Table 3. Information on the selected 100 genomic sequences [Part 3]

\begin{tabular}{|c|c|c|c|c|}
\hline ID & $\begin{array}{l}\text { Genome } \\
\text { size }\end{array}$ & Description & Lineage & Source \\
\hline CU928173 & 1114666 & Zygosaccharomyces rouxii strain CBS732 chr. A complete sequence. & Eukaryota, Fungi & $\mathrm{EBI}$ \\
\hline DG000010 & 27390870 & Oryzias latipes DNA, chr.10, strain: HdrR. & Eukaryota, Metazoa & $\mathrm{EBI}$ \\
\hline FA000001 & 10049037 & Drosophila melanogaster unordered unlocalized genomic scaffolds (chrUn) & Eukaryota, Metazoa & $\mathrm{EBI}$ \\
\hline FM178379 & 3325165 & Aliivibrio salmonicida LFI1238 chr. 1 & $\begin{array}{l}\text { Bacteria, } \\
\text { Proteobacteria }\end{array}$ & $\mathrm{EBI}$ \\
\hline FN543502 & 5346659 & Citrobacter rodentium ICC168, & $\begin{array}{l}\text { Bacteria, } \\
\text { Proteobacteria }\end{array}$ & $\mathrm{EB} \mid$ \\
\hline FN554974 & 4531609 & Trypanosoma brucei gambiense DAL972 chr. 11, complete sequence & Eukaryota, Euglenozoa & $\mathrm{EBI}$ \\
\hline FO082874 & 3568623 & Babesia microti strain RI chr. III, complete sequence. & Eukaryota, Alveolata & $\mathrm{EBI}$ \\
\hline FP929060 & 3108859 & Clostridiales sp. SM4/1 draft genome. & Bacteria, Firmicutes & $\mathrm{EBI}$ \\
\hline FR798980 & 512965 & Leishmania braziliensis MHOM/BR/75/M2904, chr. 6 & Eukaryota, Euglenozoa & $\mathrm{EBI}$ \\
\hline $\begin{array}{l}\text { GCA } \\
000002035.2\end{array}$ & 60348388 & Danio rerio genome assembly, chr1 & Eukaryota, Metazoa & $\mathrm{EBI}$ \\
\hline $\begin{array}{l}\text { GCA } \\
000151905.1\end{array}$ & 229507203 & Gorilla gorGor3.1 chr. 1 & Eukaryota & Ensembl \\
\hline HE601630 & 9743550 & Schistosoma mansoni strain Puerto Rico chr. 7, & Eukaryota, Metazoa & $\mathrm{EBI}$ \\
\hline HE616744 & 1292049 & Torulaspora delbrueckii CBS 1146 chr. 3, & Eukaryota, Fungi & $\mathrm{EBI}$ \\
\hline HE616749 & 833973 & Torulaspora delbrueckii CBS 1146 chr. 8, & Eukaryota, Fungi & $\mathrm{EBI}$ \\
\hline HE806319 & 1449145 & Tetrapisispora blattae CBS 6284 chr. 4, & Eukaryota, Fungi & $\mathrm{EBI}$ \\
\hline HE978314 & 1290777 & Kazachstania naganishii CBS 8797 chr. 1, & Eukaryota, Fungi & $\mathrm{EBI}$ \\
\hline NC 003070.9 & 30427671 & Arabidopsis thaliana chr. 1, complete sequence. & $\begin{array}{l}\text { Eukaryota, } \\
\text { Viridiplantae }\end{array}$ & $\mathrm{NCBI}$ \\
\hline NC 007605 & 171823 & Human herpesvirus 4 complete wild type genome. & Viruses, dsDNA viruses & $\mathrm{NCBI}$ \\
\hline NC 008394.4 & 45064769 & $\begin{array}{l}\text { Oryza sativa Japonica Group DNA, chr. 1, complete sequence, cultivar: } \\
\text { Nipponbare }\end{array}$ & $\begin{array}{l}\text { Eukaryota, } \\
\text { Viridiplantae }\end{array}$ & $\mathrm{NCBI}$ \\
\hline
\end{tabular}


Table 3. Information on the selected 100 genomic sequences [Part 3] (Continued)

\begin{tabular}{|c|c|c|c|c|}
\hline NC 008397.2 & 30039014 & $\begin{array}{l}\text { Oryza sativa Japonica Group DNA, chr. 4, complete sequence, cultivar: } \\
\text { Nipponbare }\end{array}$ & $\begin{array}{l}\text { Eukaryota, } \\
\text { Viridiplantae }\end{array}$ & $\mathrm{NCBI}$ \\
\hline NC 008398.2 & 32124789 & $\begin{array}{l}\text { Oryza sativa Japonica Group DNA, chr. 5, complete sequence, cultivar: } \\
\text { Nipponbare }\end{array}$ & $\begin{array}{l}\text { Eukaryota, } \\
\text { Viridiplantae }\end{array}$ & $\mathrm{NCBI}$ \\
\hline NC 008399.2 & 30357780 & $\begin{array}{l}\text { Oryza sativa Japonica Group DNA, chr. 6, complete sequence, cultivar: Nippon } \\
\text { bare }\end{array}$ & $\begin{array}{l}\text { Eukaryota, } \\
\text { Viridiplantae }\end{array}$ & $\mathrm{NCBI}$ \\
\hline NC 008400.2 & 28530027 & $\begin{array}{l}\text { Oryza sativa Japonica Group DNA, chr. 7, complete sequence, cultivar: } \\
\text { Nipponbare }\end{array}$ & $\begin{array}{l}\text { Eukaryota, } \\
\text { Viridiplantae }\end{array}$ & $\mathrm{NCBI}$ \\
\hline NC 008401.2 & 23661561 & $\begin{array}{l}\text { Oryza sativa Japonica Group DNA, chr. 8, complete sequence, cultivar: } \\
\text { Nipponbare }\end{array}$ & $\begin{array}{l}\text { Eukaryota, } \\
\text { Viridiplantae }\end{array}$ & $\mathrm{NCBI}$ \\
\hline NC 008403.2 & 35571569 & $\begin{array}{l}\text { Oryza sativa Japonica Group DNA, chr. 10, complete sequence, cultivar: } \\
\text { Nipponbare }\end{array}$ & $\begin{array}{l}\text { Eukaryota, } \\
\text { Viridiplantae }\end{array}$ & $\mathrm{NCBI}$ \\
\hline NC 008467.1 & 35863200 & Populus trichocarpa linkage group I, whole genome shotgun sequence & $\begin{array}{l}\text { Eukaryota, } \\
\text { Viridiplantae }\end{array}$ & $\mathrm{NCBI}$ \\
\hline NT 024477.14 & 1034903 & Homo sapiens chr. 12 genomic contig, GRCh37.p13 Primary Assembly & Eukaryota, Metazoa & $\mathrm{NCBI}$ \\
\hline NT 024498.12 & 369930 & $\begin{array}{l}\text { Homo sapiens chr. } 13 \text { genomic contig, } \\
\text { GRCh37.p13 Primary Assembly }\end{array}$ & Eukaryota, Metazoa & $\mathrm{NCBI}$ \\
\hline NT 029928.13 & 3915179 & Homo sapiens chr. 3 genomic contig, GRCh37.p13 Primary Assembly & Eukaryota, Metazoa & $\mathrm{NCBI}$ \\
\hline NT 077528.2 & 556644 & Homo sapiens chr. 7 genomic contig, GRCh37.p13 Primary Assembly & Eukaryota, Metazoa & $\mathrm{NCBI}$ \\
\hline NT 078094.2 & 868660 & Homo sapiens chr. 15 genomic contig, GRCh37.p13 Primary Assembly & Eukaryota, Metazoa & $\mathrm{NCBI}$ \\
\hline NT 167185.1 & 3353625 & Homo sapiens chr. 1 genomic contig, GRCh37.p13 Primary Assembly & Eukaryota, Metazoa & $\mathrm{NCBI}$ \\
\hline NT 167196.1 & 754004 & Homo sapiens chr. $\times$ genomic contig, GRCh37.p13 Primary Assembly & Eukaryota, Metazoa & NCBI \\
\hline
\end{tabular}

Based on running time, Masai, SOAP2 and SeqAlto were among the fastest.

In terms of precision and recall, the average performance over 100 genomic sequences for read lengths 50 , 75 and 100 is summarized in Table 4. All aligners were generally very accurate and increasingly so at longer read lengths. On average, CUSHAW2, Masai, and Smalt performed consistently well across read lengths 50-100, whereas Bowtie2, BWA-SW and SeqAlto performed equally well at read lengths 75-100, but were slightly inferior at read length 50. Strictly based on numbers, SHRiMP2 had very good accuracy (in terms of precision and recall), but for larger genomes, it ran very slow. Performance of GASSST seemed peculiar with some recall values larger than 1 . This is possible if a read is aligned to multiple locations by the aligner and counted

Table 4 Precision and recall averaged across 100 genomes at read lengths 50, 75, 100.

\begin{tabular}{lllllll}
\hline & Prec-50 & Rec-50 & Prec-75 & Rec-75 & Prec-100 & Rec-100 \\
\hline Bowtie2 & 0.9871 & 0.9062 & 0.9943 & 0.9721 & 0.9965 & 0.9891 \\
BWA-SW & 0.9886 & 0.8983 & 0.9952 & 0.9831 & 0.9972 & 0.9951 \\
CUSHAW2 & 0.9882 & 0.9868 & 0.9956 & 0.9956 & 0.9975 & 0.9975 \\
GASSST & 0.9836 & 1.1109 & 0.9897 & 1.0339 & 0.9914 & 0.9757 \\
Masai & 0.9889 & 0.9861 & 0.9958 & 0.9903 & 0.9976 & 0.9790 \\
mrFAST & 0.9408 & 0.5700 & 0.9862 & 0.9166 & 0.9833 & 0.9268 \\
SeqAlto & 0.9875 & 0.8851 & 0.9956 & 0.9748 & 0.9976 & 0.9925 \\
SHRiMP2 & 0.9892 & 0.9798 & 0.9958 & 0.9905 & 0.9975 & 0.9974 \\
Smalt & 0.9858 & 0.9714 & 0.9954 & 0.9944 & 0.9974 & 0.9974 \\
SOAP2 & 0.9893 & 0.9025 & 0.9959 & 0.7904 & 0.9976 & 0.6526 \\
\hline
\end{tabular}

as correct more than once by the SAMtool evaluation package, which allows a gap (default value of 20) between predicted and actual read positions.

In brief, many of these aligners (e.g. Bowtie2, CUSHAW2, SeqAlto) performed similarly accurately on the tested 100 genomes. Without additional information, it can be difficult to decide between these high-performing aligners. It would be useful if we could predict how accurately they perform on new genomes. To explore the aligners' performance on new genomes, we will examine the correlation between various measures of genome complexity and alignment accuracy.

\section{Correlation between genome complexity and alignment performance}

Our experiments showed that an appropriate choice of length-sensitive measure of complexity correlated highly with short-read performance of most aligners across read lengths, rates of mutation and sequencing error. Figures 2, 3 , and 4 show the correlation between complexity measures $D_{k}, R_{k}, D, I$ and alignment performance (precision and recall) at read lengths 50,75 , and 100 , respectively, for the 100 genomes. We see that the $D$-complexity surprisingly had no correlation to performance across all aligners. The I-complexity (Becher et al. [12]) had better but still very low correlation, with correlation coefficients between 0 and -0.3 .

We can see that there is a value of $k$ for which $D_{k}$ that correlated highly with performance for both precision and recall, across all read lengths of 50,75, and 100. For most aligners, the correlation coefficients were approximately 


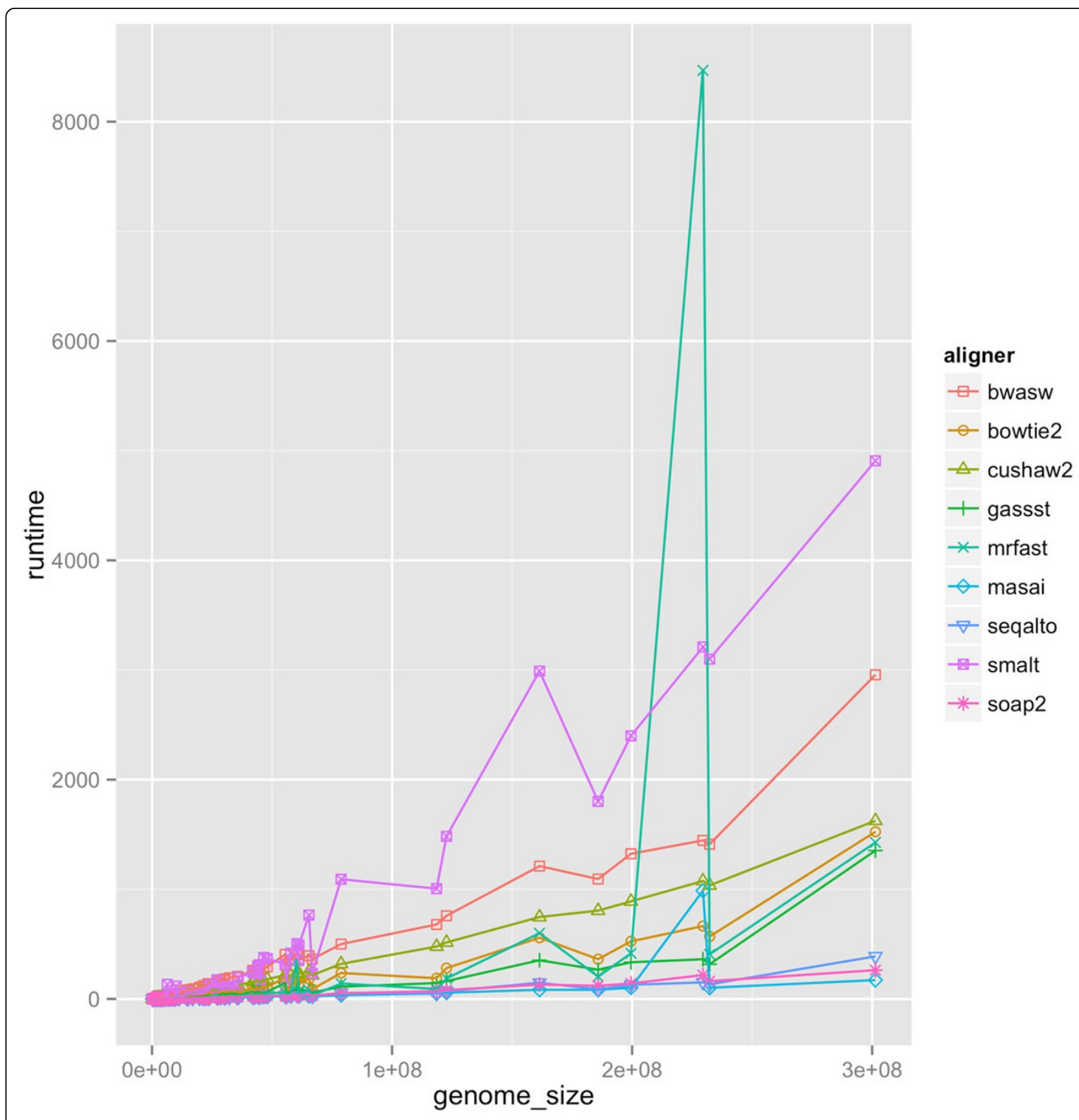

Figure 1 Running time (in seconds) of aligners as function of genome size with $2 x$ coverage, read length equal to 100 , sequencing error at $2 \%$, mutation rate at $0.1 \%$.

0.95. The only noticeable exception is for GASSST whose correlation coefficients were comparatively lower than those of the others. We think the explanation for this is in GASSST's peculiar performance as we reported earlier, whereby its recalls were above 1 for many of the 100 genomes. Additionally, we could see that when recalls were comparative lower for mrFAST and BWA-SW at read length 50, their correlations were also comparative lower than the other aligners'. It is important to note that some aligners were designed to work optimally with longer reads and consequently do not work very well with shorter reads. One can conclude that if aligners perform predictably in their comfort zones, $D_{k}$ (or $R_{k}$ ), is a good complexity measure that correlates highly to the accuracy of aligning reads to genomes.

A close examination of the results shows that the value of $k$ for which $D_{k}$ correlated highest with performance was very close to the read length. For example, 


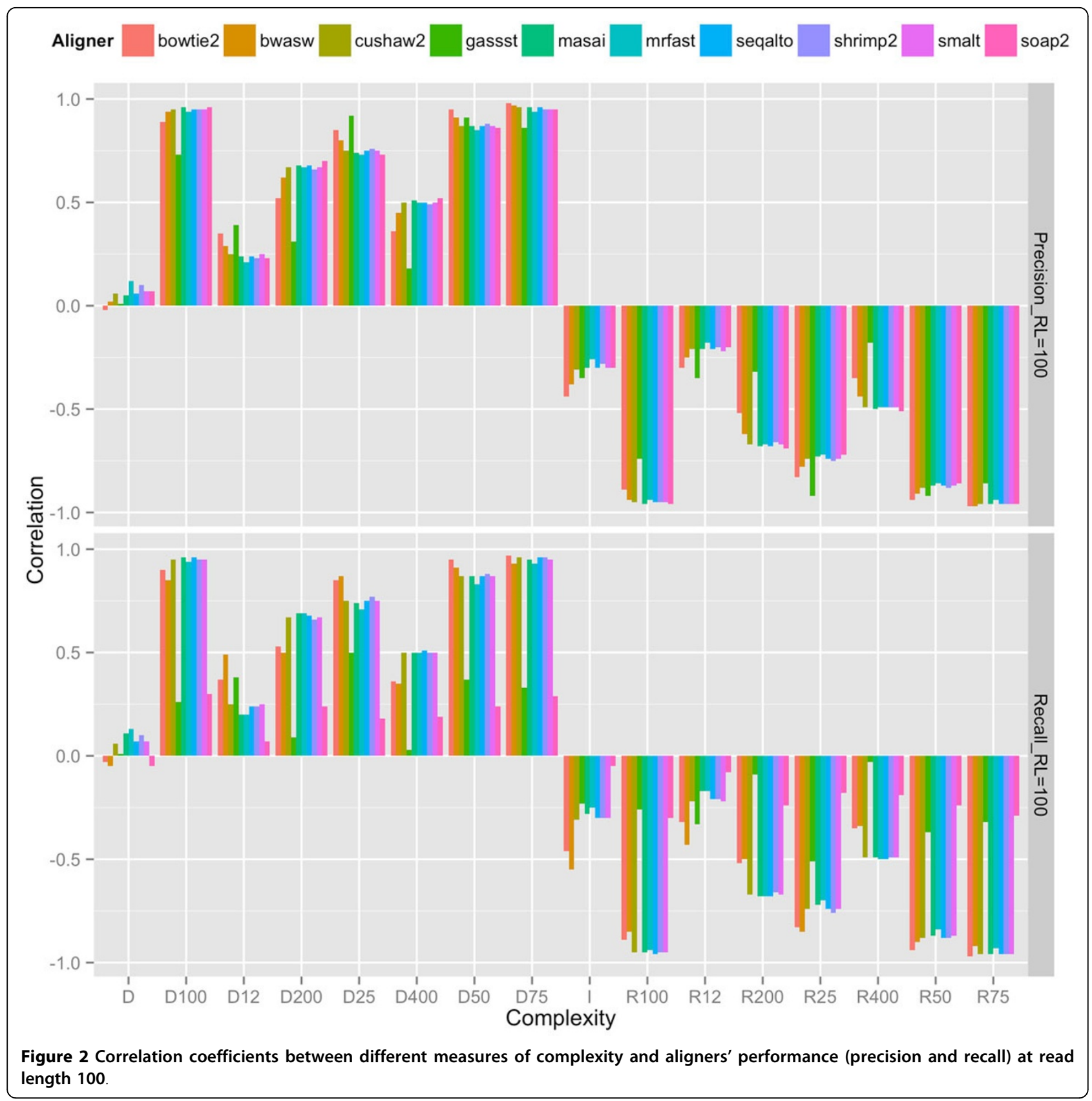

at read length $100, D_{100}$ had the highest correlations across aligners; at both read lengths 50 and $75, D_{50}$ had the highest correlations, although $D_{25}$ also had very high correlations at read length 50 . Thus, the most accurate measure of complexity to understand the difficulty of short-read alignment is length sensitive. Intuitively, this is because repeats of length close to 75 , for example, influence the accuracy of the alignment of reads of length 75 .

The fact that the best value of $k$ is less than or equal to read length, and not larger than it implies that $D_{k}$ accounts for approximate repeats. To see this, observe that a 75-mer repeat might not be part of a 100-mer, but surely contains several 50 -mer repeats (26 of them, to be precise). This means that $D_{100}$ neglects to account for several 50-mers, whereas $D_{50}$ accounts for all of these, and these 50-mer repeats directly have an influence of the accuracy of aligning reads of length 75 . This is probably why $D_{50}$ had a better correlation profile to complexity than $D_{100}$ did. The fact that $D_{k}$ accounts for approximate repeats longer than $k$ can be explained formally by the so-called q-gram lemma, which states that 


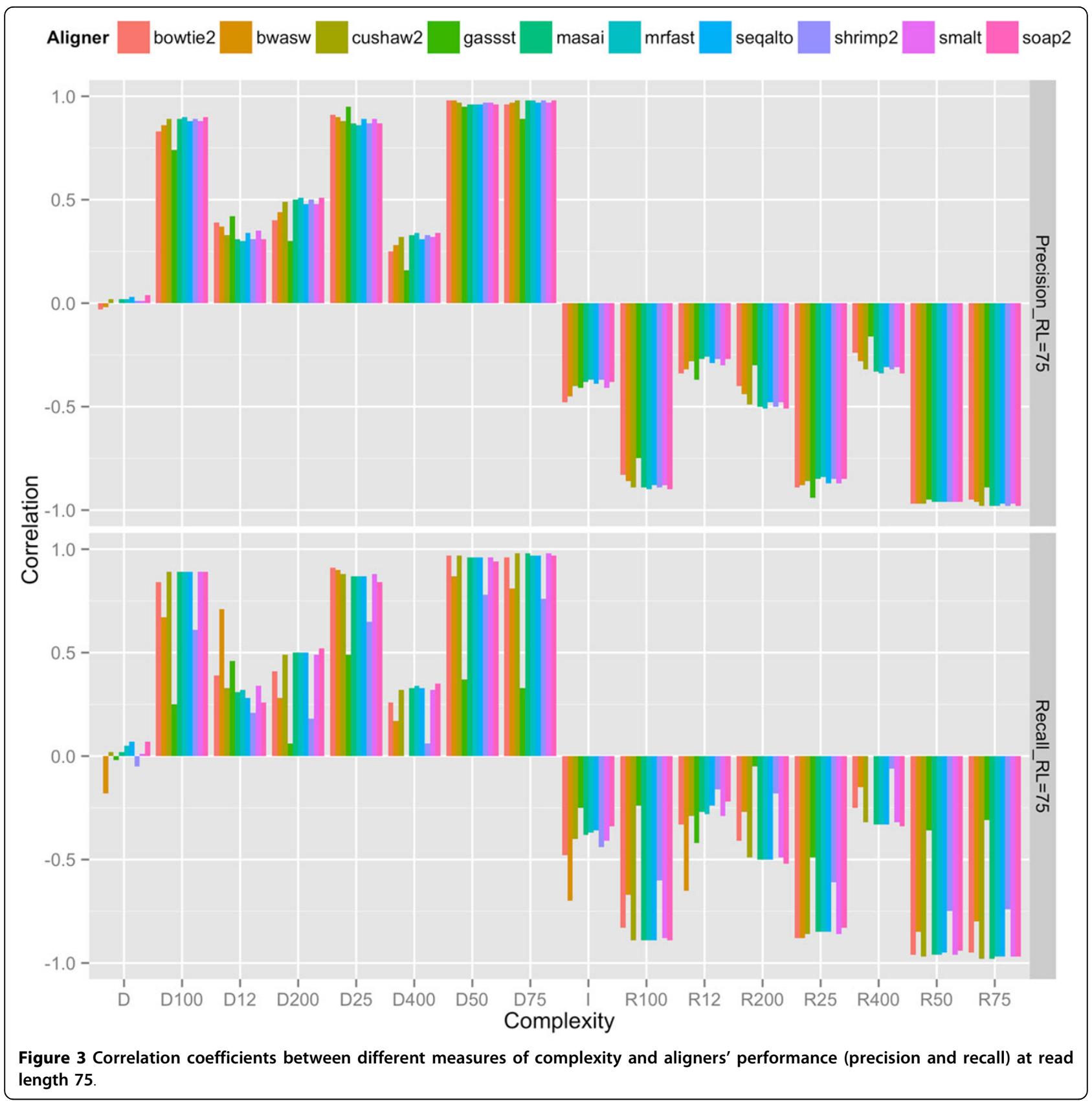

two sequences of length $k$ with edit distance $e$ share at least $n-q+1-q e q$-grams. An estimate of complexity involving counting approximate repeats might give better correlation. However, the computing of approximate repeats is computational expensive compared to linear time computation of $D_{k}$. The best computation of approximate repeats we know of using a lossless filter [19] has an average running time of $O\left(n^{2}\right)$. For long genomes, this running time is not desirable. Since $D_{k}$ and $R_{k}$ already correlated quite highly (approximately 0.95 ) for many aligners, a more efficient running time (linear instead of quadratic) seemed to be a better trade-off than a potentially better correlation.

At different rates of sequencing error and mutation, respectively, we observed similarly high correlation between performance of the aligners and length-sensitive measures of complexity. To study the correlation at different rates of sequencing error and mutation rates, respectively, we chose to correlate $D_{100}$ and alignment performance on reads of length 100 . This case is representative for the correlation between the most appropriate length-sensitive measure and aligners' performance at 


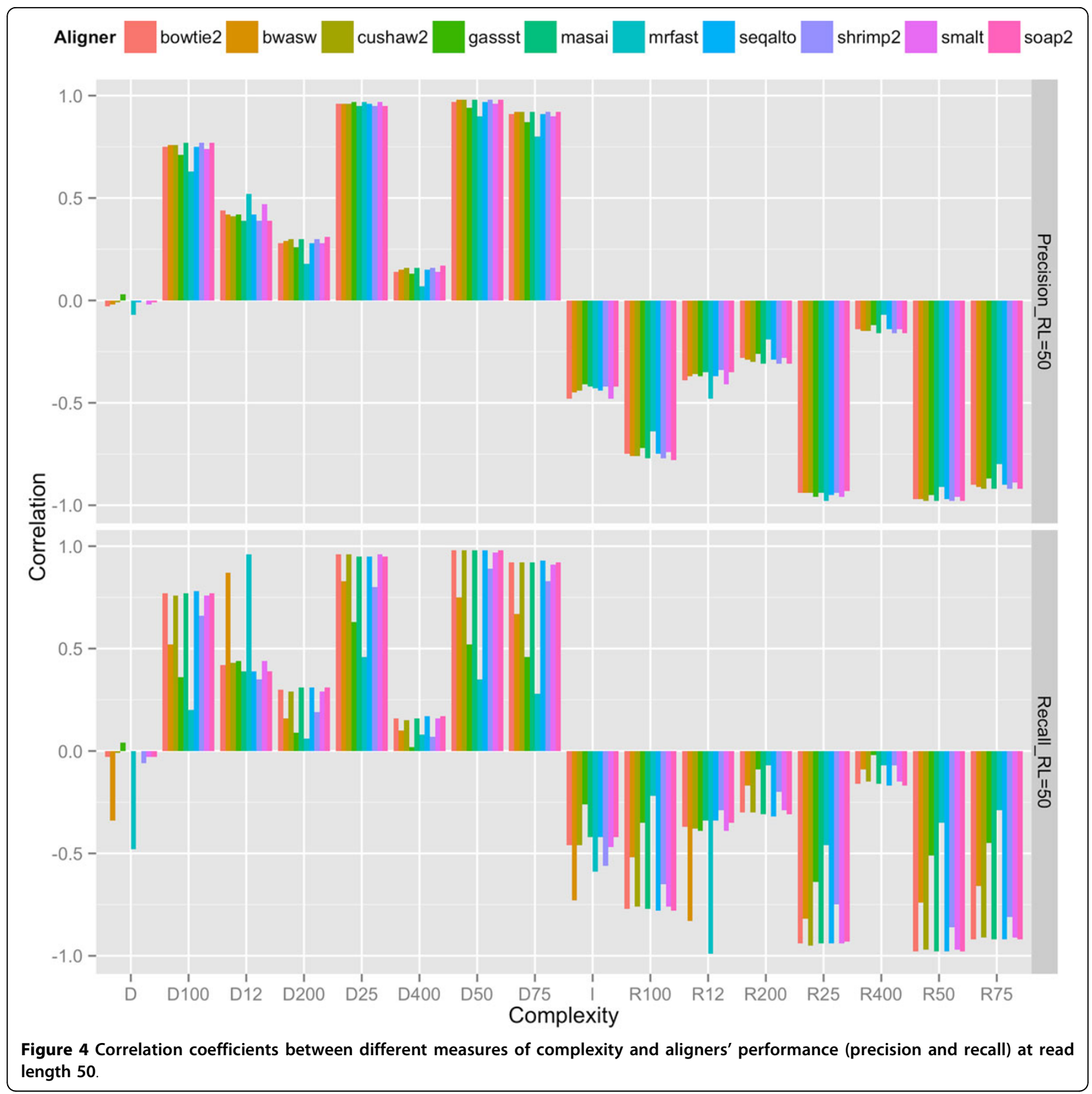

a given read length. Figure 5 and 6 show the correlation between $D_{100}$ and aligners' performance of aligning reads of length 100 at different sequencing error rates and different mutation rates, respectively. Across all rates of sequencing error and mutation, the correlation between precision of all aligners and $D_{100}$ ranged from high to very high. The lowest correlation was obtained for GASSST at about 0.75 . Correlations for the other aligners were around 0.95. Similarly, the correlation between recall and $D 100$ was also high for almost all aligners. Overall, compared to precision, recall was, however, not as highly correlated. This might be explained by some aligners' conservative strategies, which aim to make few false positive alignments at the expense of making more false negatives. Further, as expected, at higher rates of sequencing error and mutation, respectively, correlation between performance and complexity decreased. Although, this decrease in correlation is affected more by increasing sequencing errors and by increasing mutations.

\section{Predicting aligner performance for unknown genomes}

The existence of many short-read aligners makes it challenging for researchers to pick the best one for their genomes of interest. Surveys such as [20] compared 


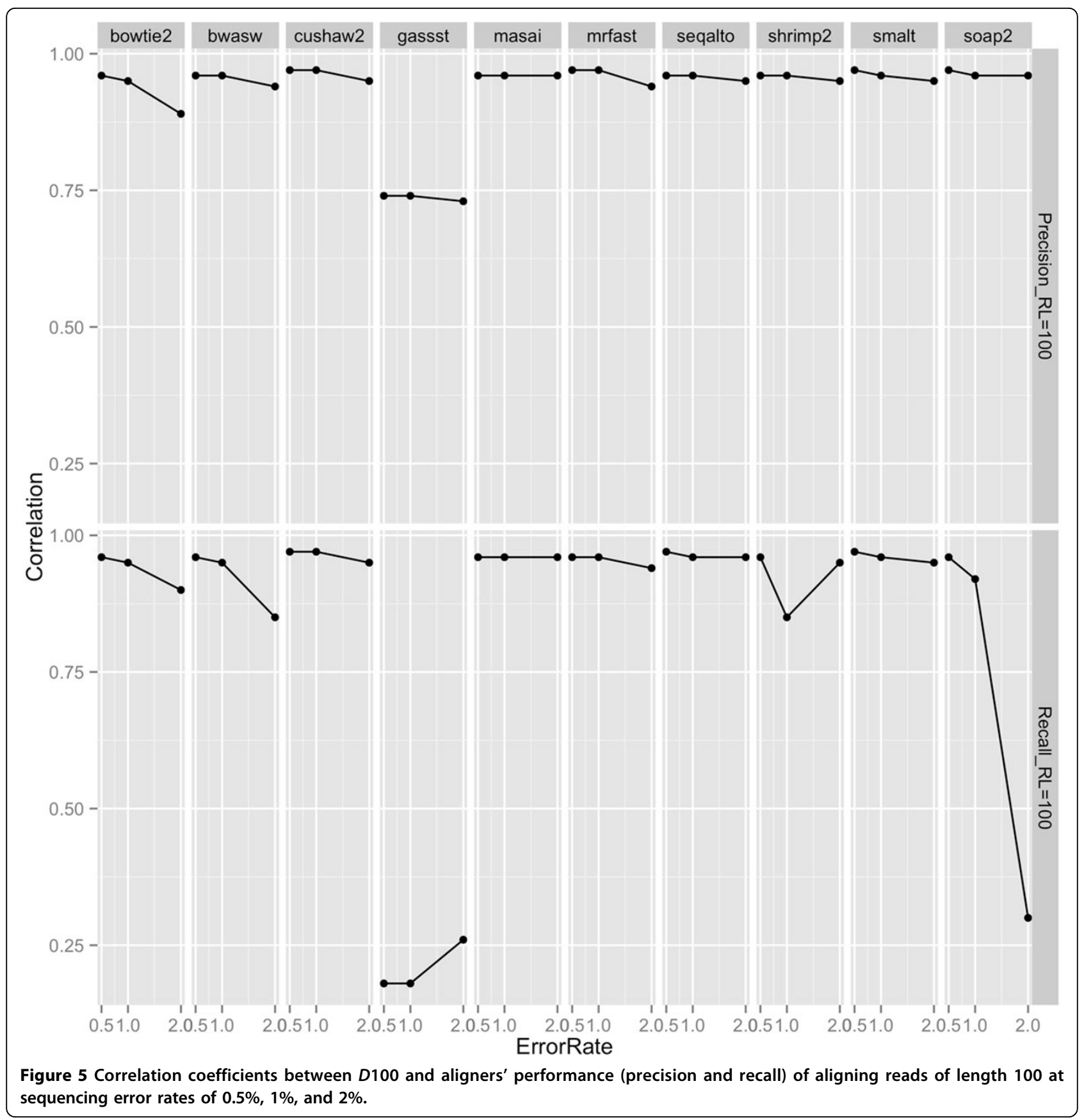

popular software packages on a few known genomes and served as a good starting point. But when it comes to adopt a particular software package, the decision seems to be a mixture of many factors including the authors' reputation, past familiarity with the software, its alignment accuracy, its quality and ease of use, its resource usage (running time and RAM), and recommendations of fellow researchers. Our focus is on accuracy, defined in terms of precision and recall.

To predict accuracy of a particular aligner on unknown genomes, researchers currently rely on its accuracy on known genomes. Such prediction can be based on summary statistics such as the top figure in Figure 7. This figure shows precisions and recalls of the aligners across 100 genomes in a boxplot figure, which shows medians, interquartile ranges among other statistics. Considering both statistics on precision and recall, we can see that with the exception of MrFast and SOAP2 (and maybe GASSST), the rest of the aligners had similar precisions and recalls across the 100 genomes. While the aligners' performance appeared similar on known genomes, what remains uncertain is, however, how well they perform on new genomes. 


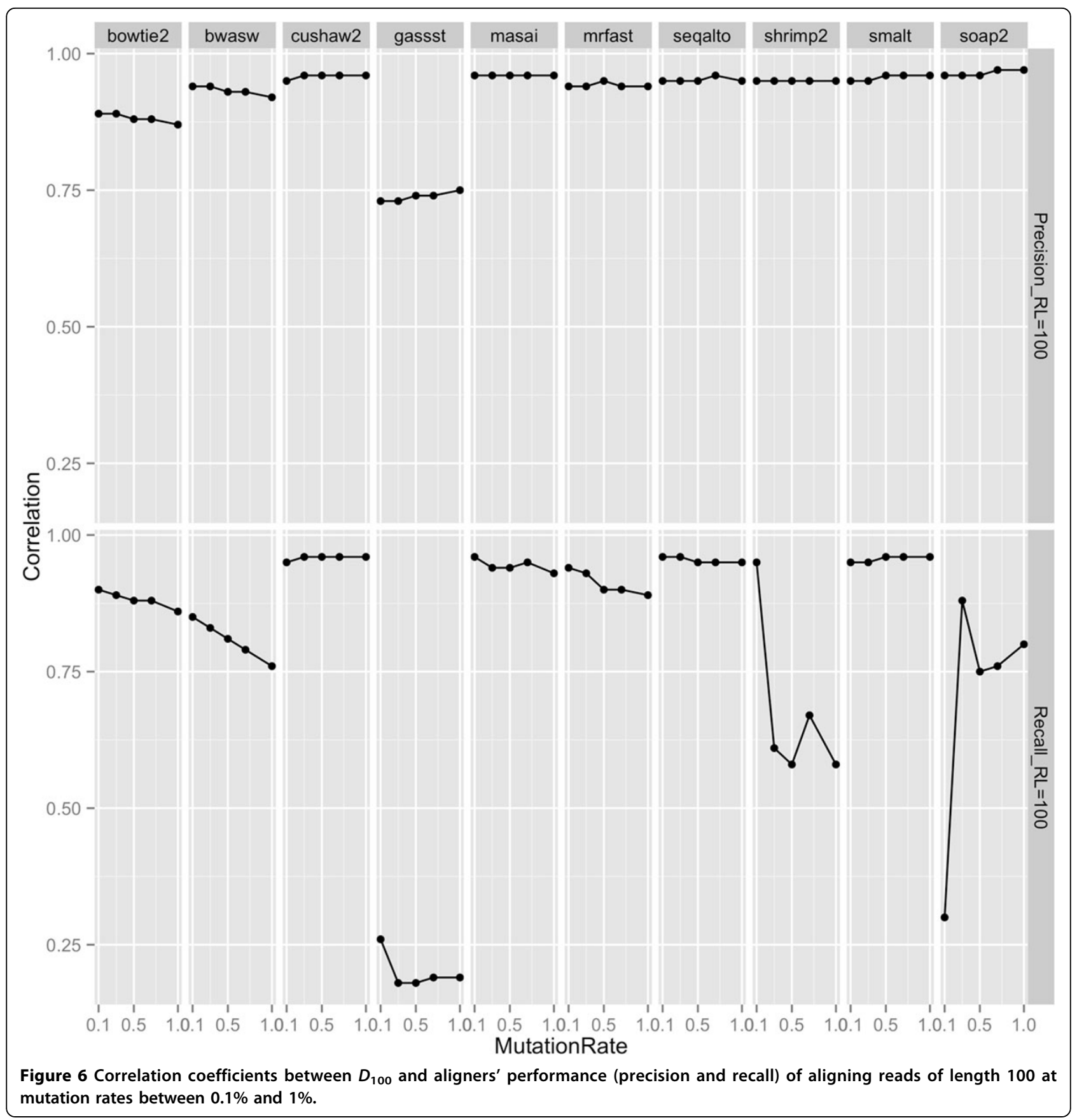

To remove this uncertainty and make more informed decisions, we might additionally incorporate correlation between genome complexity and accuracy. To illustrate this strategy, consider the bottom figure in Figure 7 shows the correlation between $D_{100}$ (since it is the best at read length 100) of the aligners' precision and recall across the 100 genomes. Comparing the performance of the high-performing aligners identified in the previous step (those other than MrFast and SOAP2), we can see that they have different levels of correlations. For instance, Bowtie2 had noticeably lower correlations (0.89 for precision and 0.90 for recall) than CUSHAW2 for both precision and recall). Thus, although Bowtie 2 and CUSHAW2 had similar accuracies for the 100 genomes, we expect that CUSHAW2 will more likely have similar accuracies for unknown genomes.

\section{The effect of $k$ on $D_{k}$ and $R_{k}$}

Measures $D_{k}$ and $R_{k}$ are length specific and may have different characteristics for different values of $k$. Figure 8 

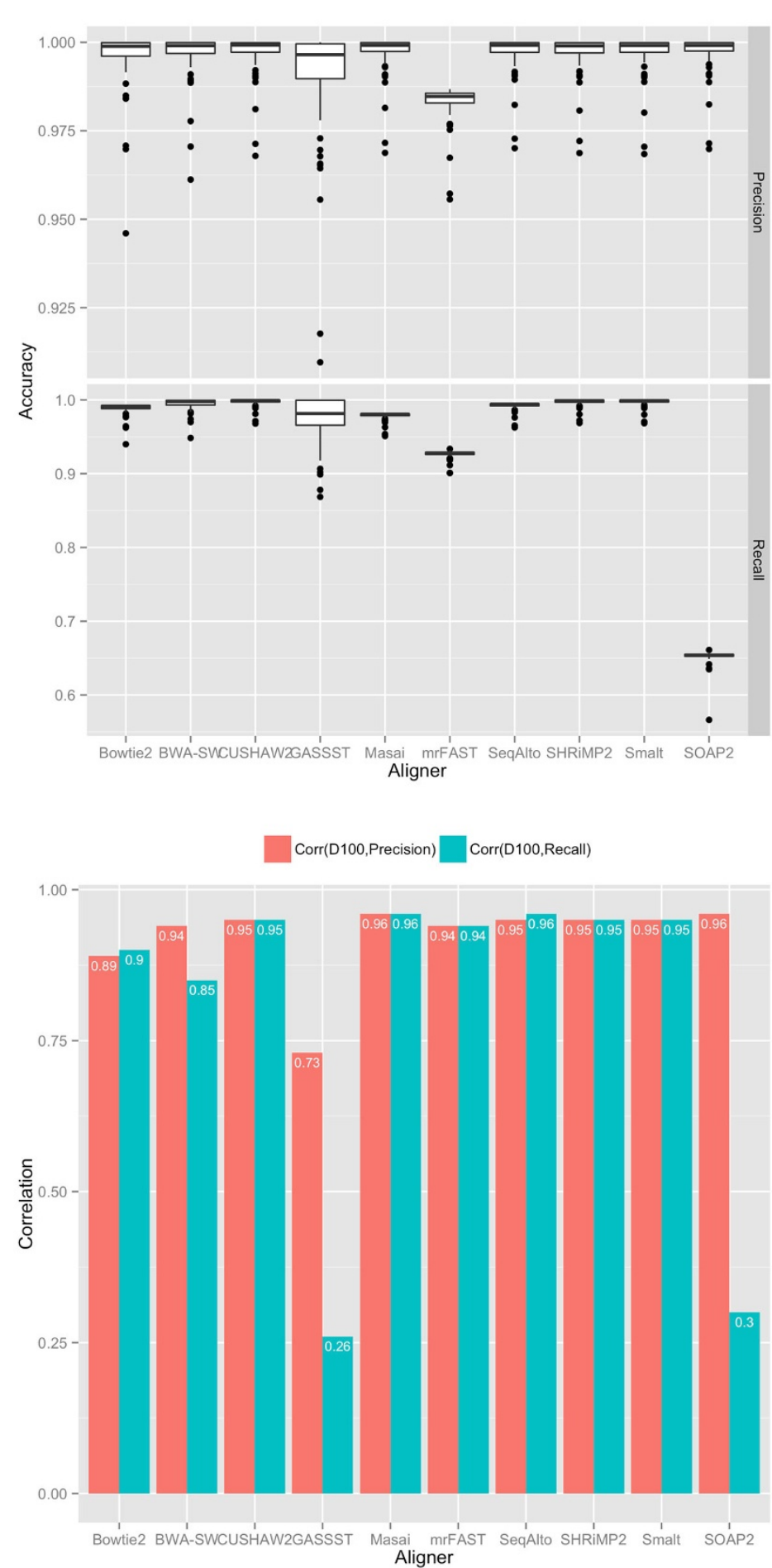

Figure 7 Top Figure: box plots of accuracy (precision and recall) of aligners across 100 genomes; read length equal to 100 . Bottom Figure: correlation between performance and $D_{100}$.

shows the cumulative distributions of $D_{k}$ and $R_{k}$ with $k=$ $12,25,50,100$. We can see that the distributions of $D_{k}$ and $R_{k}$ are similar, but in an opposite fashion. For $D_{12}$ or $R_{12}$, the distribution of complexity of the 100 genomes is quite uniform across the range from 0 to 1 . With $k>12$, however, the distribution is quite non-uniform. As $k$ becomes larger, the distribution of $D_{k}$ (or $R_{k}$ ) becomes much more concentrated toward 1 (or 0 ). 


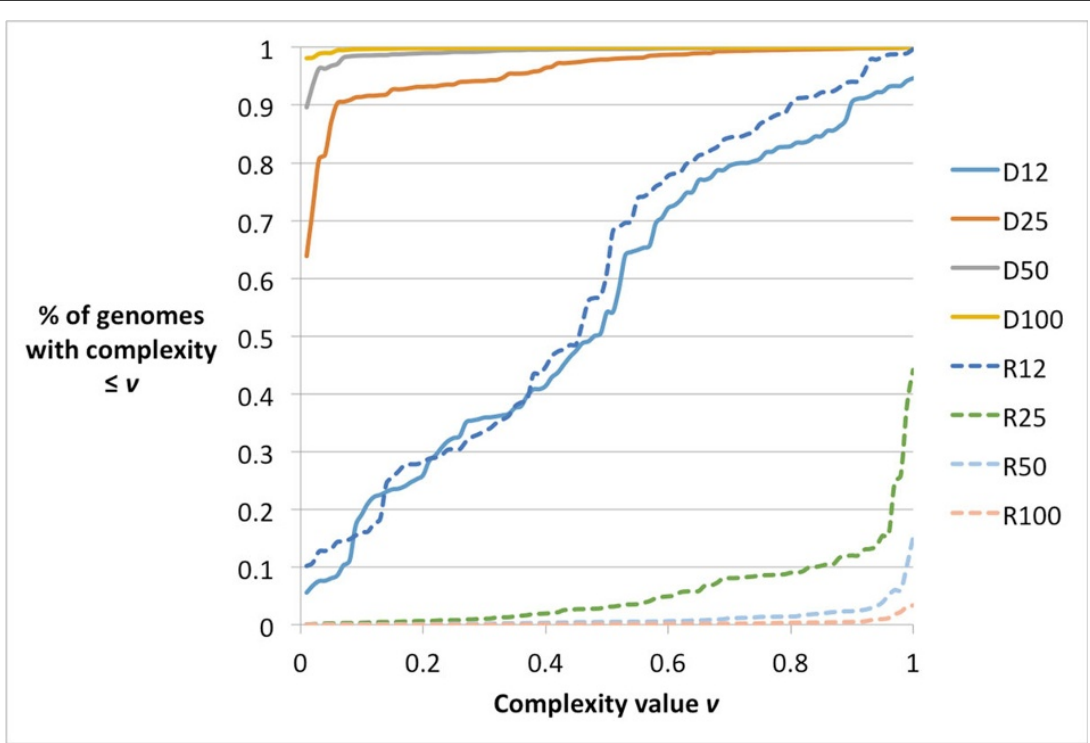

Figure 8 Cumulative distributions of $D_{k}$ and $R_{k}(k=12,25,50,100)$ for 100 genomes.

The transition from near-uniform distributions of $D_{12}$ (and $R_{12}$ ) to very skewed distributions of $D_{k}$ (and $R_{k}$ ), for $k \geq 50$, might explain for the low correlation of $D_{12}$ (and $R_{12}$ ) to alignment accuracy. Thus, we can stipulate that right choice of $k$ is essential for correlating complexity and alignment accuracy. The right choice of $k$ appears to be similar to read length as we have observed.

\section{Conclusions}

We demonstrated that length sensitive measures were suitable for studying how genome complexity affected the of short-read alignment. This work has implications for theoretical studies of genome complexity, as well as for comparing alignment methods, and designing costeffective experiments to assemble genomes. Beyond short-read alignment, these measures should be useful for problems such as short-read assembly, which are affected by genomic repeats.

This method depends on the simulation of reads with known alignment locations, from which we can compute the number of correctly aligned reads for the calculation of precision and recall. With real reads, we cannot know this information. Better simulation of reads will improve the predictive power of this method.

\section{Competing interests}

The authors declare that they have no competing interests.

\section{Authors' contributions}

VP designed methods, experiments, evaluations. SG, NSV, QT selected data, aligners and performed experiments.

\section{Acknowledgements}

This work is partly supported by NSF grant CCF-1320297.

\section{Declarations}

Publication charges for this work were funded by NSF grant CCF-1320297 to VP.

This article has been published as part of BMC Bioinformatics Volume 16 Supplement 17, 2015: Selected articles from the Fourth IEEE International Conference on Computational Advances in Bio and medical Sciences (ICCABS 2014): Bioinformatics. The full contents of the supplement are available online at http://www.biomedcentral.com/bmcbioinformatics/ supplements/16/S17.

Published: 7 December 2015

\section{References}

1. David M, Dzamba M, Lister D, llie L, Brudno M: SHRiMP2: sensitive yet practical short read mapping. Bioinformatics 2011, 27(7):1011-1012.

2. Alkan C, Kidd JM, Marques-Bonet T, Aksay G, Antonacci F, et al: Personalized copy number and segmental duplication maps using nextgeneration sequencing. Nat Genet 2009, 41(10):1061-1067.

3. Mu JC, Jiang H, Kiani A, Mohiyuddin M, Asadi NB, Wong WH: Fast and accurate read alignment for resequencing. Bioinformatics 2012, 28(18):2366-2373.

4. Rizk G, Lavenier D: GASSST: global alignment short sequence search tool. Bioinformatics 2010, 26(20):2534-2540.

5. Langmead B, Salzberg SL: Fast gapped-read alignment with bowtie 2 . Nat Methods 2012, 9(4):357-359.

6. Li H, Durbin R: Fast and accurate long-read alignment with burrowswheeler transform. Bioinformatics 2010, 26(5):589-595.

7. Li R, Li Y, Kristiansen K, Wang J: SOAP: short oligonucleotide alignment program. Bioinformatics 2008, 24(5):713-714.

8. Liu Y, Schmidt B: Long read alignment based on maximal exact match seeds. Bioinformatics 2012, 28(18):318-324.

9. Siragusa $E$, Weese $D$, Reinert K: Fast and accurate read mapping with approximate seeds and multiple backtracking. Nucleic Acids Res 2013, 41(7):e78.

10. Ponstingl H, Ning Z: SMALT-a new mapper for DNA sequencing reads. F1000 Posters 2010, 1:313.

11. Yu X, Guda K, Willis J, Veigl M, Wang Z, Markowitz MD, et al: How do alignment programs perform on sequencing data with varying qualities and from repetitive regions? BioData Min 2012, 5(1):6.

12. Becher $\mathrm{V}$, Heiber PA: A linearly computable measure of string complexity. Theoretical Computer Science 2012, 438:62-73.

13. Chor B, Horn D, Goldman N, Levy T, Massingham T: Genomic DNA k-mer spectra: models and modalities. Genome Biology 2009, 10(10):R108. 
14. Kurtz S, Narechania A, Stein JC, Ware D: A new method to compute k-mer frequencies and its application to annotate large repetitive plant genomes. BMC Genomics 2008, 9:517.

15. Whiteford NE, Haslam NJ, Weber G, Prugel-Bennett A, Essex JW, Neylon C, et al: Visualizing the repeat structure of genomic sequences. Complex Systems 2008, 17(4):381-398.

16. Kärkkäinen J, Sanders P, Burkhardt S: Linear work suffix array construction. J ACM 2006, 53(6):918-936.

17. Kasai $T$, Lee $G$, Arimura $H$, Arikawa S, Park K: Linear-time longest-commonprefix computation in suffix arrays and its applications. Proceedings of the 12th Annual Symposium on Combinatorial Pattern Matching Lecture Notes in Computer Science 2001, 181-192.

18. Li H, Handsaker B, Wysoker A, Fennell T, Ruan J, Homer N, et al: The sequence alignment/map format and samtools. Bioinformatics 2009, 25(16):2078-2079.

19. Peterlongo P, Sacomoto GA, do Lago AP, Pisanti N, Sagot MF: Lossless filter for multiple repeats with bounded edit distance. Algorithms Mol Biol 2009, 4:3.

20. Li H, Homer N: A survey of sequence alignment algorithms for nextgeneration sequencing. Briefings in Bioinformatics 2010, 11(5):473-483.

doi:10.1186/1471-2105-16-S17-S3

Cite this article as: Phan et al:: How genome complexity can explain the difficulty of aligning reads to genomes. BMC Bioinformatics 2015

16(Suppl 17):S3.

\section{Submit your next manuscript to BioMed Central} and take full advantage of:

- Convenient online submission

- Thorough peer review

- No space constraints or color figure charges

- Immediate publication on acceptance

- Inclusion in PubMed, CAS, Scopus and Google Scholar

- Research which is freely available for redistribution

Submit your manuscript at www.biomedcentral.com/submit 